\title{
Laparoscopic right hemicolectomy with complete mesocolic excision: a cadaver model
}

\author{
H. Kessler ${ }^{1} \cdot$ M. Gouvea Monteiro de Camargo ${ }^{1}$ (I) $\cdot$ C. P. Delaney ${ }^{1} \cdot$ S. R. Steele ${ }^{1}$
}

Received: 16 November 2019 / Accepted: 4 December 2019 / Published online: 4 January 2020

(c) Springer Nature Switzerland AG 2020

Complete mesocolic excision is defined as a surgical technique that comprises sharp dissection of the mesocolon's visceral plane from the retroperitoneal plane without breaching of the visceral layer, which could lead to tumor cell spread within the peritoneal cavity. Hohenberger et al. [1] from Erlangen University first described complete mesocolic excision (CME) in conjunction with central vascular ligation (CVL) and demonstrated a correlation between anatomic and mesentery-based resection of right-sided colon cancer. Since CME became standard of care, 5-year local recurrence rates at Erlangen University Hospital decreased from 6.5 to $3.6 \%$ and 5-year cancer-related survival improved from 82.1 to $89.1 \%$ [1].

Several studies have demonstrated superior long-term oncological outcomes for CME [1-3]. Due to still unsatisfying long-term results of colon cancer surgery in many institutions, standardization of quality-based surgery worldwide is needed. Training programs play an essential role in improving outcomes for colon and rectal cancer $[4,5]$.

This video shows a systematic approach to laparoscopic extended right hemicolectomy with CME in a cadaveric model, which includes preservation of the mesocolic planes, medial to lateral colon mobilization, high vascular tie and central lymph node dissection and suggests a standard approach to this technique to be used in training programs.

Electronic supplementary material The online version of this article (https://doi.org/10.1007/s10151-019-02135-2) contains supplementary material, which is available to authorized users.

\section{H. Kessler}

kessleh@ccf.org

1 Department of Colorectal Surgery, Digestive Disease and Surgery Institute, Cleveland Clinic, 9500 Euclid Avenue, A 30, Cleveland, $\mathrm{OH} 44195$, USA

\section{Compliance with ethical standards}

Conflict of interest The authors declare that they have no conflict of interest.

Ethical approval All procedures performed in studies involving human participants were in accordance with the ethical standards of the institutional and/or national research committee (Institutional Review Board of the Cleveland Clinic Foundation) and with the 1964 Helsinki declaration and its later amendments or comparable ethical standards.

Informed consent Informed consent was obtained from all individual participants included in the study.

Funding This video was not funded by any company.

\section{References}

1. Hohenberger W, Weber K, Matzel K et al (2009) Standardized surgery for colonic cancer: complete mesocolic excision and central ligation-technical notes and outcome. Color Dis 11:354-364. https://doi.org/10.1111/j.1463-1318.2008.01735.x

2. Bertelsen CA, Neuenschwander AU, Jansen JE et al (2015) Disease-free survival after complete mesocolic excision compared with conventional colon cancer surgery: a retrospective, population-based study. Lancet Oncol 16:161-168. https://doi. org/10.1016/S1470-2045(14)71168-4

3. Kotake K, Mizuguchi T, Moritani K et al (2014) Impact of D3 lymph node dissection on survival for patients with T3 and T4 colon cancer. Int J Colorectal Dis 29:847-852. https://doi. org/10.1007/s00384-014-1885-Z

4. West NP, Hohenberger W, Weber K et al (2010) Complete mesocolic excision with central vascular ligation produces an oncologically superior specimen compared with standard surgery for carcinoma of the colon. J Clin Oncol 28:272-278. https://doi. org/10.1200/JCO.2009.24.1448

5. Bertelsen CA, Bols B, Ingeholm $P$ et al (2011) Can the quality of colonic surgery be improved by standardization of surgical technique with complete mesocolic excision? Color Dis 13:11231129. https://doi.org/10.1111/j.1463-1318.2010.02474.x

Publisher's Note Springer Nature remains neutral with regard to jurisdictional claims in published maps and institutional affiliations. 